\title{
Comparison of Trimethoprim-Sulfamethoxazole vs. Clindamycin for the Treatment of Children with Musculoskeletal Infections
}

\author{
Julian Tyte, ${ }^{1}$ Kelsey Russell, ${ }^{2,3}$ James Wood ${ }^{2,3}$
}

${ }^{1}$ Indiana University School of Medicine, ${ }^{2}$ Indiana University School of Medicine, Department of Pediatrics, Ryan White Center for Pediatric Infectious Diseases and Global Health, ${ }^{3}$ Pediatric and Adolescent Comparative Effectiveness Medicine, Department of Pediatrics

\section{Background:}

Musculoskeletal infections ([MSKI]; osteomyelitis, septic arthritis, pyomyositis) are common invasive infections in children. With increasing antibiotic resistance and allergies, treatment options are limited. Trimethoprim-sulfamethoxazole (TMP-SMX) has activity against common MSKI pathogens, yet pediatric MSKI outcomes data are limited. Our aim was to evaluate outcomes of children with MSKI treated with TMP-SMX compared to clindamycin. We hypothesized that outcomes would be similar between groups.

\section{Methods:}

We conducted a retrospective review of children $\leq 18$ years old, admitted to Riley Hospital for Children from January 2010-June 2021 treated with TMP-SMX or clindamycin for MSKI.

Patients were identified by ICD-9/10 codes. Patients were excluded if TMP-SMX or clindamycin was not the main treatment, they had symptoms $>30$ days, hardware associated infection, or an alternative diagnosis. Treatment success was defined as no evidence of infection at the end therapy. Treatment failure was defined as antibiotic intolerance, or development of recurrent/chronic osteomyelitis. Adverse drug reactions were recorded.

\section{Results:}

One-hundred-sixty-three patients (30 TMP-SMX 133 clindamycin) met eligibility criteria. The majority were non-Hispanic white males, median age of 1.75 years (IQR, 1-3.75) for the TMPSMX group, and 7 years (IQR 3-10) for the clindamycin group. Osteomyelitis was the most common infection type in both groups (TMP-SMX 43.3\%, clindamycin 54.1\%). S. aureus was the most common pathogen in both groups (TMP-SMX $36.7 \%$, clindamycin $48.1 \%$ ). Of the patients that completed follow-up, $84 \%$ successfully completed treatment with TMP-SMX, compared to $86.4 \%$ with clindamycin (odds ratio [OR] 0.82; 95\% $\mathrm{Cl} 0.25-2.46, p=0.75$ ). Adverse drug reactions occurred in $6.7 \%$ of the TMP-SMX group, compared to $10.5 \%$ in the clindamycin group (OR 0.61; 95\% Cl 0.13-2.57, p=0.52).

\section{Conclusions:}

Preliminary results show that TMP-SMX is well tolerated and not associated with higher risk of treatment failure compared to clindamycin in the treatment of pediatric MSKI. Randomized controlled trials to evaluate optimal treatment are needed. 